\title{
Semaphorin 7A promotes EGFR-TKI resistance in EGFR mutant lung adenocarcinoma cells
}

Yuhei Kinehara, ${ }^{1,2,3}$ Izumi Nagatomo, ${ }^{2}$ Shohei Koyama, ${ }^{1,2,3}$ Daisuke Ito, ${ }^{1,2,3}$ Satoshi Nojima, ${ }^{1.3,4}$ Ryota Kurebayashi, ${ }^{2}$ Yoshimitsu Nakanishi, , ${ }^{1,3}$ Yasuhiko Suga, ${ }^{1,2,3}$ Yu Nishijima-Futami, ${ }^{1,2,3}$ Akio Osa, ${ }^{2}$ Takeshi Nakatani, ,2,3 Yasuhiro Kato, ,2,3 Masayuki Nishide, ${ }^{1,2,3}$ Yoshitomo Hayama, ${ }^{1,2,3}$ Masayoshi Higashiguchi, ${ }^{2}$ Osamu Morimura, ${ }^{2}$ Kotaro Miyake, ${ }^{2}$ Sujin Kang, ${ }^{1,3,5}$ Toshiyuki Minami, ${ }^{2,6}$ Haruhiko Hirata, ${ }^{2}$ Kota Iwahori, ${ }^{2}$ Takayuki Takimoto, ${ }^{2}$ Hyota Takamatsu,, ${ }^{1,2}$ Yoshito Takeda, ${ }^{2}$ Naoki Hosen, ${ }^{1,2,7}$ Shigenori Hoshino, ${ }^{8}$ Yasushi Shintani, ${ }^{9}$ Meinoshin Okumura, ${ }^{9}$ Toru Kumagai, ${ }^{10}$ Kazumi Nishino, ${ }^{10}$ Fumio Imamura, ${ }^{10}$ Shin-ichi Nakatsuka, ${ }^{11}$ Takashi Kijima, ${ }^{2,6}$ Hiroshi Kida, ${ }^{2}$ and Atsushi Kumanogoh ${ }^{1,2,3}$

'Department of Immunopathology, World Premier International Research Center, Immunology Frontier Research Center, Osaka University, Suita, Osaka, Japan. 'Department of Respiratory Medicine and Clinical Immunology, Osaka University Graduate School of Medicine, Suita, Osaka, Japan. ${ }^{3}$ The Japan Agency for Medical Research and Development-Core Research for Evolutional Science and Technology (AMED-CREST), Tokyo, Japan. ${ }^{4}$ Department of Pathology, Osaka University Graduate school of Medicine, Suita, Osaka, Japan. ${ }^{5}$ Department of Immune Regulation, Immunology Frontier Research Center, Osaka University, Suita, Osaka, Japan. ${ }^{6}$ Division of Respiratory Medicine, Department of Internal Medicine, Hyogo College of Medicine, Nishinomiya, Hyogo, Japan. ${ }^{7}$ Department of Cancer Stem Cell Biology, Osaka University Graduate School of Medicine, Suita, Osaka, Japan. ${ }^{8}$ Saito Yukoukai Hospital, Ibaraki, Osaka, Japan. ${ }^{9}$ Department of General Thoracic Surgery, Osaka University Graduate School of Medicine, Suita, Osaka, Japan. ${ }^{10}$ Department of Thoracic Oncology and "Department of Pathology, Osaka International Cancer Institute, Osaka, Osaka, Japan.

Although responses to ECFR tyrosine kinase inhibitors (EGFR-TKIs) are initially positive, 30\%-40\% of patients with EGFR-mutant tumors do not respond well to EGFR-TKIs, and most lung cancer patients harboring ECFR mutations experience relapse with resistance. Therefore, it is necessary to identify not only the mechanisms underlying EGFR-TKI resistance, but also potentially novel therapeutic targets and/or predictive biomarkers for EGFR-mutant lung adenocarcinoma. We found that the GPI-anchored protein semaphorin 7A (SEMA7A) is highly induced by the ECFR pathway, via mTOR signaling, and that expression levels of SEMA7A in human lung adenocarcinoma specimens were correlated with mTOR activation. Investigations using cell culture and animal models demonstrated that loss or overexpression of SEMA7A made cells less or more resistant to EGFRTKIs, respectively. The resistance was due to the inhibition of apoptosis by aberrant activation of ERK. The ERK signal was suppressed by knockdown of integrin $\beta 1$ (ITCB1). Furthermore, in patients with EGFR mutant tumors, higher SEMA7A expression in clinical samples predicted poorer response to EGFR-TKI treatment. Collectively, these data show that the SEMA7A-ITCB1 axis plays pivotal roles in EGFR-TKI resistance mediated by ERK activation and apoptosis inhibition. Moreover, our results reveal the potential utility of SEMA7A not only as a predictive biomarker, but also as a potentially novel therapeutic target in EGFR-mutant lung adenocarcinoma.

Conflict of interest: The authors have declared that no conflict of interest exists.

License: Copyright 2018, American Society for Clinical Investigation.

Submitted: June 25, 2018 Accepted: November 6, 2018 Published: December 20, 2018

Reference information: JCI Insight. 2018;3(24):e123093. https://doi.org/10.1172/jici. insight.123093.

\section{Introduction}

Lung cancer is the leading cause of death from cancer in both men and women (1). Non-small cell lung cancer (NSCLC) accounts for $\sim 85 \%$ of lung cancer cases (2), and in the last several years, adenocarcinoma has become the predominant type of NSCLC (3). The discovery of driver mutations has drastically changed therapeutic strategies in lung adenocarcinoma cases, and currently, the assessment of EGFR mutation status has become a mandatory step in choosing the most effective first-line treatment for patients with advanced disease (4). EGFR is mutated and constitutively active in $\sim 20 \%$ of NSCLC 
patients $(5,6)$, with a significantly higher proportion in patients with adenocarcinoma, women, patients of Asian ethnicity, and nonsmokers. Especially in East Asia, $\sim 40 \%$ of adenocarcinoma patients harbor EGFR mutations (7), $\sim 85 \%$ include exon 19 deletions or L858R substitutions. Indeed, multiple clinical trials have demonstrated that first-line administration of EGFR tyrosine kinase inhibitors (EGFRTKIs) in patients harboring EGFR mutations results in more prolonged progression-free survival (PFS) than cytotoxic chemotherapy.

Despite the good response to first-line treatment with EGFR-TKI, most patients harboring EGFR mutations experience relapse with resistance to this class of drugs, predominantly caused by the T790M secondary gatekeeper mutation, which accounts for $50 \%-60 \%$ of resistance cases $(8,9)$, or activation of an alternative pathway such as $\operatorname{HGF} / \operatorname{Met}(10,11)$. Although the prevalence of EGFR-TKI resistance observed in the clinic has increased, the mechanisms underlying resistance have not been fully determined. Notably, 30\%-40 \% of patients with EGFR-mutant (EGFR-Mut) tumors do not respond well to EGFR-TKIs, implying that EGFR mutation may not be the sole factor determining drug response (7, 12, 13). Intrinsic and acquired forms of resistance to EGFR-TKI represent critical problems to be solved. In addition, drug tolerant states have also been reported, and some of these obstacles to treatment are directly involved in driver mutation signals (14).

Semaphorins, a large phylogenetically conserved family of secreted or membrane-bound proteins, are divided into 8 classes based on sequence similarity and distinct structural features. They were originally identified as axon guidance molecules; consistent with this, abnormalities in these proteins have been implicated in the pathogenesis of neurologic disorders such as Alzheimer's disease and motor neuron degeneration (15). Semaphorins are also involved in immune responses and the regulation of organogenesis, angiogenesis, apoptosis, and neoplasia (15-17). SEMA7A, a glycosylphosphatidylinositol-anchored (GPI-anchored) glycoprotein on the plasma membrane, was first shown to be involved in olfactory nerve outgrowth (18). We and others have demonstrated that SEMA7A activates ERK signals via integrin $\beta 1$ (ITGB1) in neurons and macrophages $(15,19,20)$. Several studies using mice or human samples demonstrated that SEMA7A expression promotes mammary tumor progression, epithelial-to-mesenchymal transition (EMT), and angiogenesis (21-24). Moreover, SEMA7A promotes cell growth in oral squamous carcinoma (25), activates tumor-associated macrophages in melanoma (26), and correlates with invasiveness of a glioblastoma cell line (27). Plexin C1, a receptor for SEMA7A, inactivates cofilin and is a potential tumor suppressor in melanoma $(28,29)$. However, the pathological significance of SEMA7A in human lung adenocarcinomas remains unclear, especially in regard to acquisition of malignant phenotypes such as treatment resistance.

In this study, we show that SEMA7A is a target gene of EGFR-Mut and that its regulation is mediated by mTOR signaling. We focused on the role of SEMA7A in EGFR-Mut lung cancer. Our findings revealed that the SEMA7A-ITGB1 axis activates ERK signaling and inhibits apoptosis induced by EGFR-TKIs, thereby contributing to acquisition of EGFR-TKI resistance. Therefore, we propose that SEMA7A could be used not only as a biomarker to predict EGFR-TKI efficacy and prognosis in EGFR-Mut tumors, but also as a possible therapeutic target in EGFR-TKI-resistant lung adenocarcinoma patients.

\section{Results}

To find candidate molecules related to EGFR-TKI resistance, we established gene transfer assays using NIH3T3 cells. We generated 2 EGFR expression vectors, WT and an Ex19 deletion Mut, and established stable transfectant NIH3T3 cells expressing either WT or Mut EGFR (Figure 1A). Transcriptome analysis revealed that Sema7A expression was significantly higher in EGFR-Mut-expressing cells than in cells expressing the WT receptor (Figure 1B and Table 1; all gene expression data have been submitted to GEO, accession number GSE104494). Also upregulated were Lgr6 and Rnf183, which have been implicated in lung cancer stem-like cells and progression of colon cancer, respectively $(30,31)$. Therefore, we focused on SEMA7A. The increase in SEMA7A expression due to activation of EGFR signaling in these cells was confirmed by both flow cytometry (FCM) (Figure 1C) and quantitative PCR (qPCR) (Figure 1D). These findings indicate that oncogenic EGFR signaling can upregulate SEMA7A. Next, we investigated whether expression of SEMA7A was detectable in clinical lung adenocarcinoma samples. Some IHC samples were strongly positive for SEMA7A expression (Figure 1E). In addition, expression of SEMA7A was detectable in the CD45 ${ }^{\text {lo }}$ cell fraction from malignant pleural effusions in some cases of EGFR-Mut lung adenocarcinoma, as determined by FCM (Figure 1F), indicating that SEMA7A is 


\section{Table 1. Microarray expression profiling of NIH3T3 cells stably expressing WT or Mut EGFR}

\begin{tabular}{lc}
\hline mRNA & fold \\
Lgr6 & 103.5 \\
Rnf183 & 65.2 \\
Sema7A & 62.0
\end{tabular}

article; https://doi.org/10.1172/jci.insight.123093DS1). FCM and qPCR revealed a clear reduction in SEMA7A expression following erlotinib or osimertinib treatment (Figure 2, A and B, and Supplemental Figure 1B), with no impact on cell viability. By contrast, SEMA7A expression was not downregulated by the cytotoxic drug carboplatin (Figure 2C and Supplemental Figure 1B). In the secondary resistance mutation-positive cell line H1975 (L858R/T790M), osimertinib suppressed EGFR activation and SEMA7A expression at both the mRNA and protein levels, whereas erlotinib did not (Supplemental Figure 1, C-E). By contrast, erlotinib suppressed neither EGFR phosphorylation (Supplemental Figure 1F) nor SEMA7A expression (Figure 2D) in H441 and H292 cell lines expressing WT EGFR. Taken together, these findings suggest that SEMA7A expression is regulated by aberrantly activated EGFR signaling in EGFR-Mut-expressing lung adenocarcinoma cells.

We then investigated the expression of SEMA7A in various human lung cancer cells (Supplemental Figure 2, A and B). SEMA7A levels tended to be higher in EGFR mutation-positive lung cancer cells than in EGFR mutation-negative cells (Supplemental Figure 2C). When we stimulated EGFR WT cells with recombinant EGF, expression of SEMA7A was upregulated following EGFR phosphorylation (Figure 2E and Supplemental Figure 2D), implying that SEMA7A can be upregulated by strong EGFR signals (either Mut EGFR or WT EGFR). Indeed, in clinical specimens, expression of SEMA7A was not correlated with EGFR mutation status (Table 2 and Figure 2F; Spearman's rank-order correlation, $P=0.86$ ), suggesting that, in clinical situations, aberrant or strong EGFR signals trigger or strengthen the expression of SEMA7A.

Activated EGFR stimulates downstream oncogenic signaling pathways, including the PI3K/AKT/ mTOR and RAS/RAF/MEK/ERK pathways, thereby promoting cell survival and proliferation (32, 33). To identify the signaling pathway responsible for regulation of SEMA7A expression, we evaluated the effects of mTOR inhibitors (rapamycin or torin) and a MEK inhibitor (U0126) on SEMA7A expression. As shown in Supplemental Figures 3, A and B, we confirmed that S6 kinase phosphorylation was downregulated by rapamycin and torin, and ERK phosphorylation was downregulated by U0126. FCM and qPCR analyses revealed a clear reduction in SEMA7A expression in cells treated with mTOR inhibitors, with no effects on cell viability (Figure 3, A-C, and Supplemental Figure 3C). By contrast, MEK inhibitor did not decrease SEMA7A levels in these cells (Figure 3D). These findings indicate that mTOR signaling is primarily responsible for SEMA7A expression downstream of EGFR mutations. To confirm the in vitro findings, we investigated the correlation between SEMA7A expression and activation of mTOR signaling in clinical samples. To this end, we performed IHC to evaluate specimens from the 190 lung adenocarcinoma patients described above; 55 samples were excluded because of material insufficiency. In the 135 patients assessed (Table 3), SEMA7A expression was correlated with S6 phosphorylation (Figure 3, E and F; Spearman's rank-order correlation, $P<0.05$ ). These results suggest that mTOR signaling regulates expression of SEMA7A in human lung adenocarcinoma, consistent with the results obtained in cell culture experiments

To clarify the pathological role of SEMA7A in EGFR mutated cells, especially with respect to EGFR-TKI sensitivity, we generated SEMA7A KO cell lines (HCC827 and HCC3255) using the CRISPR/Cas9 system (Supplemental Figure 4, A-D). To establish erlotinib-resistant sublines, we cultured SEMA7A WT and KO cells in the presence of erlotinib. Treatment was initiated at $2.5 \mathrm{nM}$ erlotinib, below the $\mathrm{IC}_{50}$, and the concentration was then increased in a stepwise manner. Before treatment, the efficacy of erlotinib did not differ between SEMA7A WT and KO cells. However, over the course of the 
A

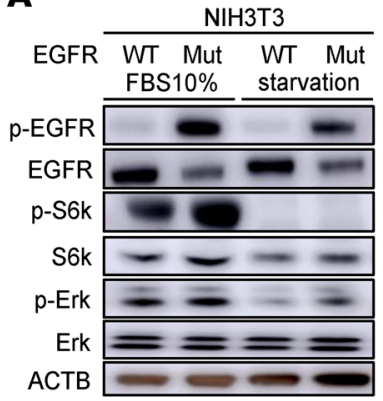

B

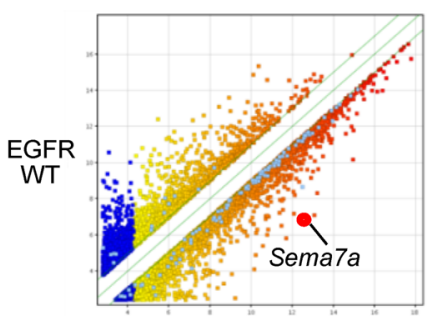

EGFR Mut
C

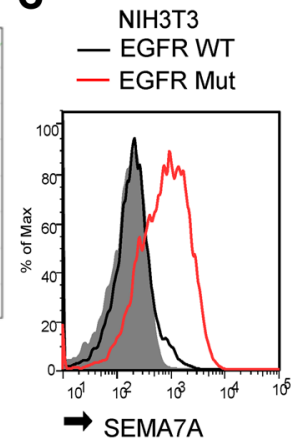

$\mathbf{F}$

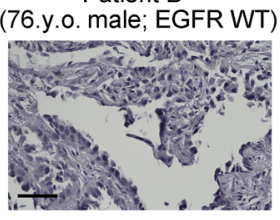

Patient B
E (70.y.o.female; EGFR Mut)

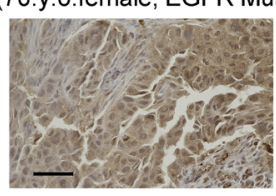

Patient 1

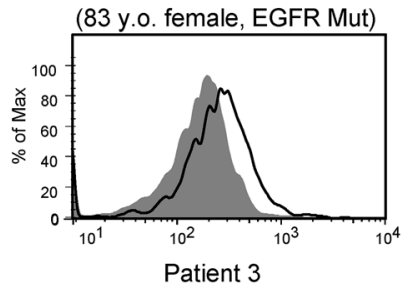

(68 y.o. female, EML4/ALK)

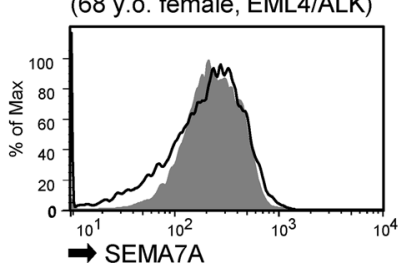

SEMA7A MFI/ iso MFI

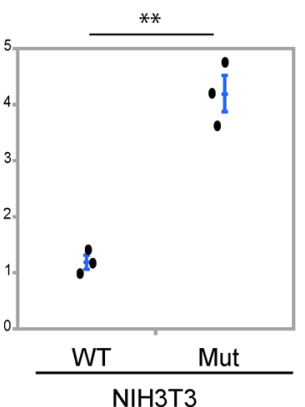

D

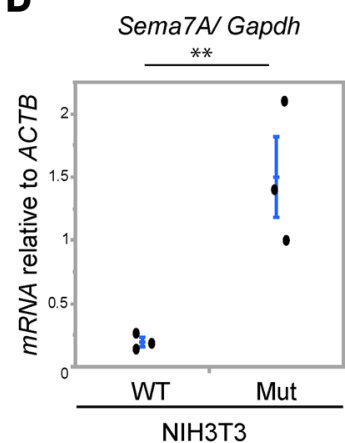

Patient 2

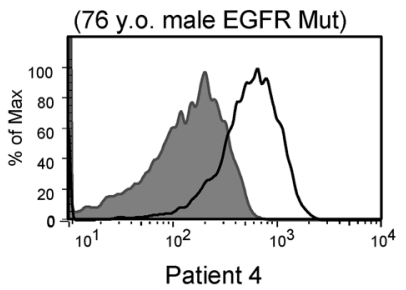

(76 y.o. male, EGFR WT)

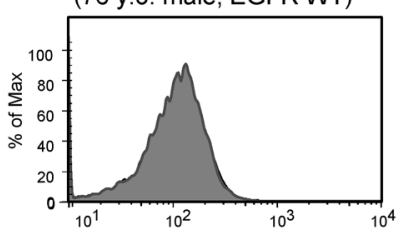

Figure 1. Oncogenic EGFR signals induce SEMA7A expression in mouse fibroblast cells, and SEMA7A is expressed in human lung adenocarcinomas. (A) Representative immunoblots showing that p-EGFR and p-S6K are upregulated in medium containing $10 \%$ FBS. Under starvation conditions, $\mathrm{p}$-Erk level is higher in EGFR-Mut than in WT EGFR-NIH3T3 cells. Data are representative of 3 independent experiments. (B) Microarray expression profiling of NIH3T3 cells stably expressing WT or Mut EGFR. (C) Confirmation of SEMA7A upregulation by FCM in EGFR-Mut-NIH3T3 cells. Left, representative of 3 independent experiments. Right, data (means $\pm \mathrm{SE}$ ) are representative of 3 independent experiments. The 2 -sample $t$ test was used to evaluate significance. ${ }^{*} P$ $<0.01$. MFI, median fluorescence intensity; iso, isotype control. Gray area indicates the isotype control. (D) qPCR confirming upregulation of Sema7A in EGFR-Mut-NIH3T3 cells. Data (means \pm SE) are representative of 3 independent experiments. The 2 -sample $t$ test was used to evaluate significance. ${ }^{*} P$ $<0.01$. (E) SEMA7A IHC of human lung adenocarcinoma samples. Scale bar: $50 \mu \mathrm{m}$. (F) SEMA7A FCM of CD45 ${ }^{\text {lo }}$ cells in malignant pleural effusions associated with human lung adenocarcinoma. Gray areas indicate isotype control.

treatment, SEMA7A KO cells were more sensitive to erlotinib than WT cells (Figure 4A and Supplemental Figure 5A). Likewise, to establish osimertinib-resistant sublines, we cultured SEMA7A WT and KO HCC 827 and H3255 cells in the presence of osimertinib. Treatment was initiated at $2.5 \mathrm{nM}$ osimertinib, below the $\mathrm{IC}_{50}$, and the concentration was then increased in a stepwise manner. Before treatment, the efficacy of osimertinib did not differ between SEMA7A WT and KO cells. After treatment, however, the SEMA7A KO HCC827 and H3255 cells were more sensitive to osimertinib than WT cells (Supplemental Figure 5B), consistent with the results obtained with erlotinib. Because expression of SEMA7A was downregulated by EGFR-TKI (Figure 2 and Supplemental Figure 1), it is possible that erlotinib treatment has an influence on the expression of SEMA7A in SEMA7A WT cells. We examined the chronological change in SEMA7A expression in WT cells during EGFR-TKI treatment and found that its expression was reduced but was maintained at a certain level (Supplemental Figure 6), suggesting that the remaining SEMA7A contributed to TKI resistance. To corroborate the involvement of SEMA7A in EGFR-TKI resistance, we generated SEMA7A-overexpressing (OE) cell lines (II-18 and H3255) using retroviral vectors (Supplemental Figure 7A). II-18 and H3255 (SEMA7A OE) and control (WT) cells were generated by transduction with the SEMA7A-expressing and the empty vector, respectively. To establish erlotinib-resistant sublines, we cultured SEMA7A WT or -OE cells derived from II-18 and $\mathrm{H} 3255$ in the presence of erlotinib $\left(10 \mathrm{nM}\right.$ and $2.5 \mathrm{nM}$, respectively, below the $\mathrm{IC}_{50}$ in each parental cell line). Before treatment, the efficacy of the EGFR-TKI did not differ between SEMA7A WT and SEMA7A OE cells, consistent with the results of the KO experiments. After treatment, however, the 
A

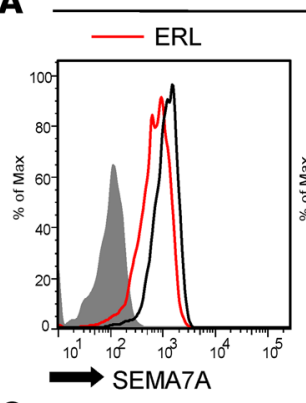

C

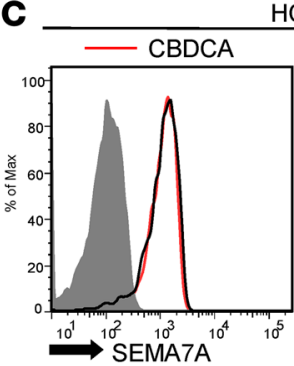

HCC827
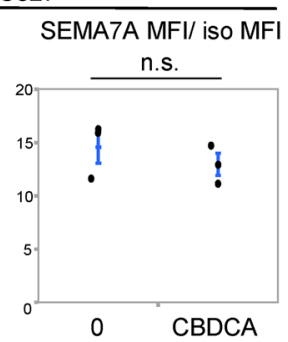

E

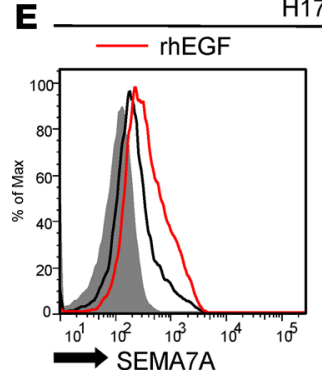

$\mathrm{HCC} 827$
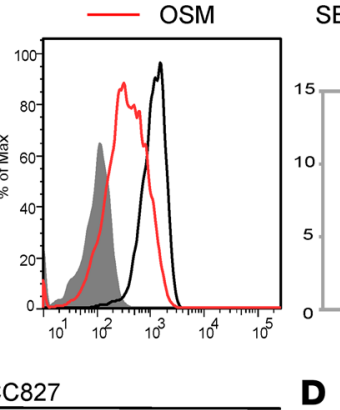

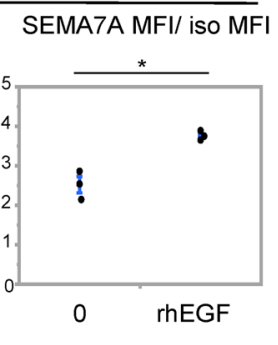

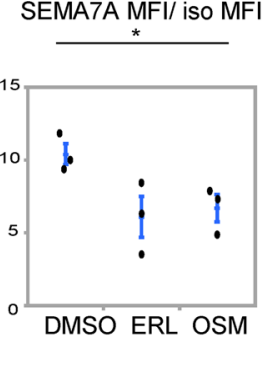

SEMA7A MFI/ iso MFI

B

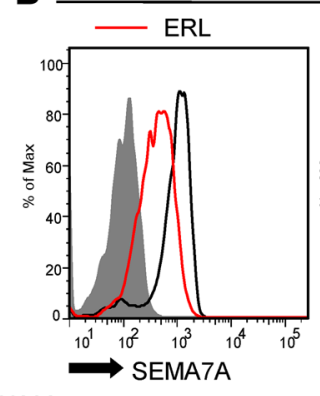

H292

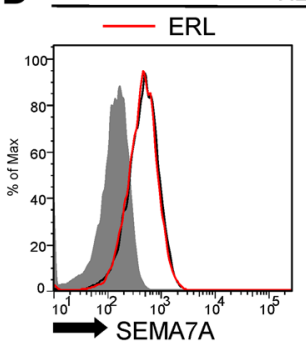

SEMA7A MFI/ iso MF

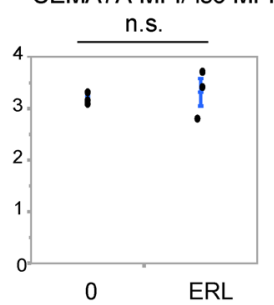

ERL

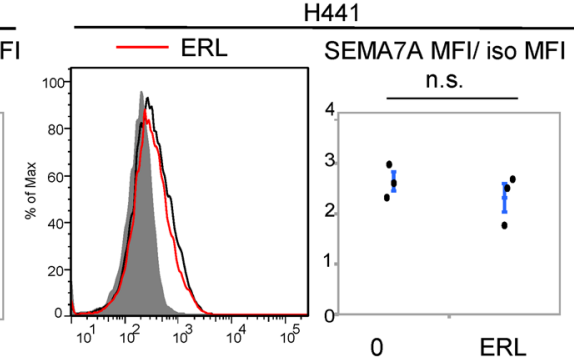

H3255

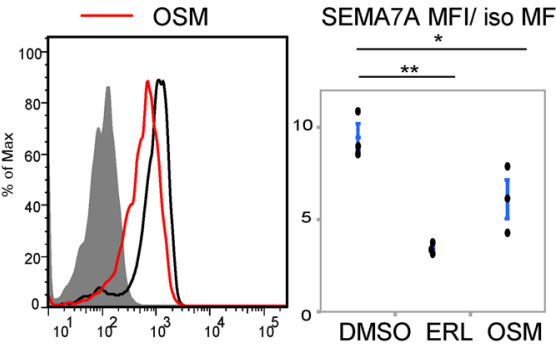

Figure 2. EGFR signals regulate SEMA7A expression in lung adenocarcinoma cells. (A-C) Reduced expression of SEMA7A in EGFR-Mut lung cancer cell lines 24 hours after treatment with erlotinib (ERL; $10 \mathrm{nM}$ ) or osimertinib (OSM; $15 \mathrm{nM}$ ). Carboplatin (CBDCA; $20 \mu \mathrm{M})$ was used as a reference. (D) Unchanged expression of SEMA7A in WT EGFR lung cancer cell lines 24 hours after treatment with erlotinib (10 nM). (E) Upregulation of SEMA7A in a WT EGFR lung cancer cell line 24 hours after treatment with recombinant human EGF (rhEGF; $10 \mathrm{nM}$ ). MFI, median fluorescence intensity; iso, isotype control. Gray area indicates the isotype control. FCM data are representative of 3 independent experiments. Data in dot plots (means \pm SE) are representative of 3 independent experiments. To evaluate significance, ANOVA followed by Dunnett's multiple comparison test was used for $\mathbf{A}$ and $\mathbf{B}$, or the 2 -sample $t$ test was used for C-E. ${ }^{*} P<0.05,{ }^{*} P<0.01$. (F) Correlation between SEMA7A expression (IHC score) and EGFR mutation status in clinical samples. Spearman's rank-order correlation was used.

SEMA7A OE cells were less sensitive to erlotinib than the WT cells (Supplemental Figure 7, B and C), while maintaining high SEMA7A expression (Supplemental Figure 7D). These data support the idea that SEMA7A contributes to EGFR-TKI resistance. The $\mathrm{IC}_{50}$ values of each EGFR-TKI in each cell line, both before and after long-term treatment, are listed in Supplemental Figure 8.

We then conducted mouse xenograft studies to confirm the results obtained in cell culture experiments. Tumorigenic capacity was equivalent between WT and KO xenografts (Figure 4B, day 30, PBS group), suggesting that SEMA7A expression was not involved in tumorigenicity per se. We then focused our interest on the relationship between SEMA7A expression and EGFR-TKI sensitivity. The initial antitumor effect of erlotinib did not differ between WT and KO xenografts (Figure 4B, erlotinib group). However, after longer treatment with erlotinib, the WT xenografts were less responsive to erlotinib than the KO xenografts (Figure 4B, $\sim$ day 50, erlotinib group). FCM exhibited SEMA7A expression in xenografts resected 3 weeks after inoculation with erlotinib (Supplemental Figure 9). These results suggest that SEMA7A contributes to development of EGFR-TKI resistance in lung cancer cells harboring EGFR mutations.

To elucidate the precise mechanisms underlying SEMA7A function, we evaluated apoptosis, the predominant mechanism of TKI-induced cell death. Before long-term treatment, erlotinib-induced apoptosis assays yielded similar results in SEMA7A WT and KO HCC827 and H3255 cells (Figure 4C and Supplemental Figure 10A). After long-term treatment with erlotinib, however, the rate of apoptosis 
Table 2. Correlations between the driver mutation status and clinical features.

\begin{tabular}{lcccc}
\hline Driver mutation status & $\begin{array}{c}\text { EGFR-Mut (-) } \\
\text { ALK fusion (-) }\end{array}$ & EGFR-Mut (+) & ALK fusion (+) & Significance \\
Sex (male/female) & $33 / 7$ & $17 / 28$ & $1 / 4$ & P.5. \\
Age (Mean; range) & $66.8(45-83)$ & $69.3(39-90)$ & $63.2(37-86)$ & \\
Brinkman index (Mean; range) & $559(0-2400)$ & $172(0-2000)$ & $240(0-1200)$ & n. \\
Stage (Mean; range) & $3.2(1-4)$ & $2.8(1-4)$ & $2.8(1-4)$ &
\end{tabular}

To evaluate significance, Fisher's exact test was used for sex. To evaluate significance, Kruskal-Wallis test followed by Steel-Dwass test was used for age, Brinkman index, and stage.

increased to a greater extent in SEMA7A KO HCC827 and H3255 cells than in WT cells (Figure 4C and Supplemental Figure 10A). By contrast, the rate of apoptosis was lower in SEMA7A OE H3255 cells than in WT cells (Supplemental Figure 10B). These results imply that SEMA7A induces TKI resistance by suppressing apoptosis.

Previous studies have reported that the major mechanisms of acquired resistance to EGFR-TKI are occurrence of the secondary T790M gatekeeper mutation (9) and amplification of the MET gene $(10,11)$. After treatment, however, we detected neither the secondary T790M mutation (Supplemental Figure 10C) nor MET overexpression (Figure 5A and Supplemental Figure 10D) in HCC827 and H3255 cells, although we did detect the T790M mutation in H1975 cells, used as a positive control (Supplemental Figure 10C). Furthermore, we did not observe either HGF overexpression or EMT in our models (data not shown). These findings suggested an alternative mechanism for resistance to EGFR-TKI in our models. We previously reported that SEMA7A activates ERK signals via ITGB1 in macrophages (19, 20). Hence, we asked whether SEMA7A could activate the ERK pathway in cancer cells. Before long-term treatment, erlotinib-induced downregulation of EGFR and ERK phosphorylation yielded similar results in SEMA7A WT and KO HCC827 and H3255 cells (Figure 5B and Supplemental Figure 10E). By contrast, after long-term treatment with erlotinib, ERK phosphorylation decreased to a greater extent in SEMA7A KO HCC827 and H3255 cells than in WT cells, although there was no difference in EGFR or AKT phosphorylation (Figure 5B and Supplemental Figure 10E). Furthermore, after long-term treatment with erlotinib, ERK phosphorylation increased to a greater extent in SEMA7A OE H3255 and II-18 cells than in WT cells, again with no difference in EGFR and AKT phosphorylation (Supplemental Figure 11). Taken together, these findings indicate that SEMA7A-mediated inhibition of apoptosis and promotion of EGFR-TKI resistance are cell-autonomous phenotypes in both cell culture and xenograft models.

As already mentioned, we and others demonstrated that SEMA7A activates ERK signals via ITGB1 in neurons and macrophages $(15,19,20)$. ITGB1 expression was verified by IHC in our EGFRMut lung adenocarcinoma samples (Supplemental Figure 12). Therefore, we investigated whether the SEMA7A-ITGB1 axis activated ERK phosphorylation in human lung adenocarcinoma cell lines. To this end, we generated ITGB1-knockdown cells using shRNA targeting ITGB1 in SEMA7A WT and KO HCC827 cells (Figure 6, A and B) and compared the effects of erlotinib on ERK signaling in those cells after long-term treatment with erlotinib. When ITGB1 was knocked down, ERK phosphorylation was clearly suppressed in SEMA7A WT cells (Figure 6C). By contrast, in SEMA7A KO cells, we observed impaired phosphorylation of ERK, regardless of whether ITGB1 was knocked down (Figure 6C). These results confirmed that the SEMA7A-ITGB1 axis is important for activating ERK, which promotes cell survival. To further confirm the importance of this axis, we examined the combinational effects of EGFR-TKI and MEK inhibitors in EGFR-Mut cells. As shown in Figure 6D, SEMA7A WT cells were more sensitive to combination than EGFR-TKI alone. By contrast, SEMA7A KO cells did not exhibit significant differences in response to the combination of EGFR-TKI and MEK inhibitors vs. EGFR-TKI alone (Figure 6D). These results support the importance of the SEMA7A-ITGB1ERK axis in resistance mechanisms.

The results described above imply that SEMA7A is a candidate predictive biomarker of EGFRTKI efficacy. Therefore, we investigated the correlation between SEMA7A expression and EGFR-TKI efficacy of patients with stage IV lung adenocarcinoma. To validate the anti-SEMA7A antibody used for IHC, SEMA7A expression was verified in SEMA7A WT or KO xenografts resected 2 weeks after 
A

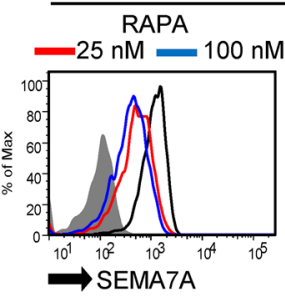

HCC827

c

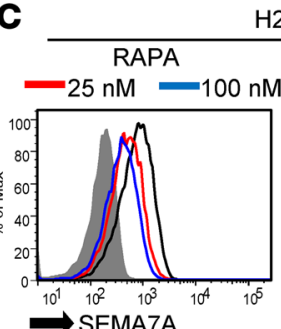

$\mathrm{H} 292$

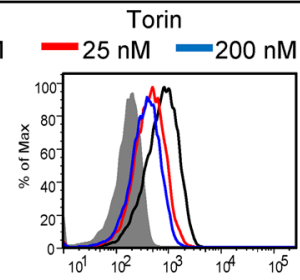

$\frac{* * *}{* * *}$

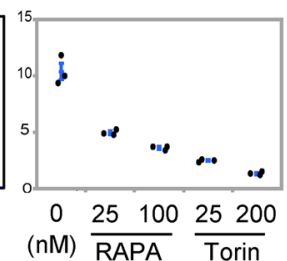

SEMA7A MFI/ iso MFI

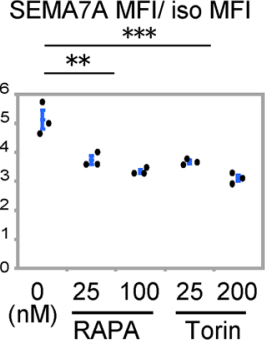

B

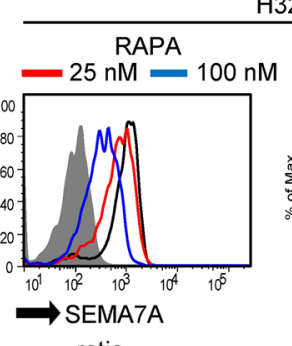

H3255

F ratio

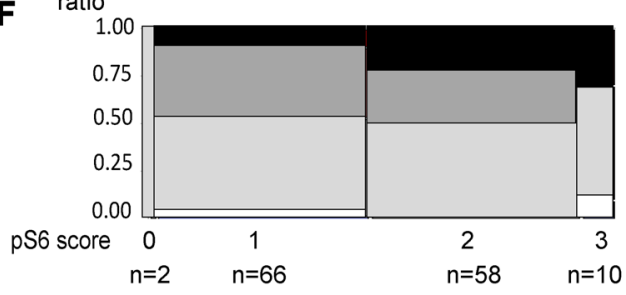

0
$(\mathrm{nM})$$\frac{25 \quad 100}{\text { RAPA }} \frac{25 \quad 200}{\text { Torin }}$

SEMA7A score

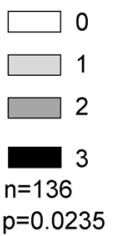

D
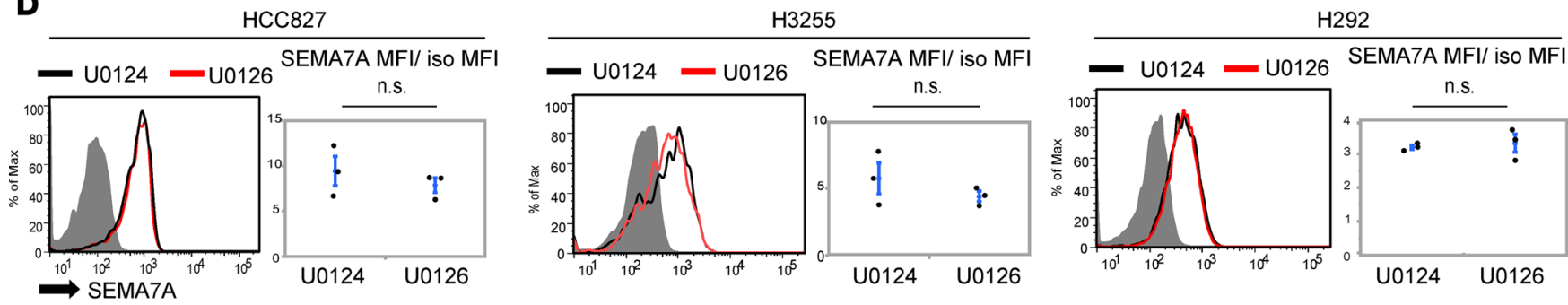

E

Patient A
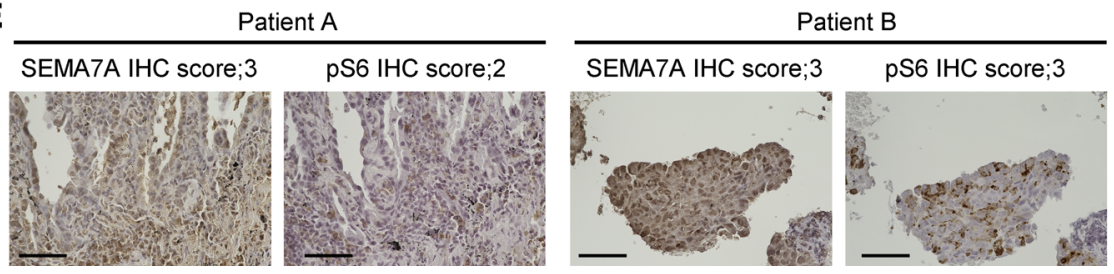

Figure 3. Inhibition of mTOR signals decreases expression of SEMA7A in human lung adenocarcinoma cell lines, regardless of EGFR mutation status. (A-C) Reduced expression of SEMA7A in both Mut and WT EGFR lung cancer cell lines 24 hours after treatment with rapamycin (RAPA) or torin at the indicated concentration. (D) Unchanged expression of SEMA7A in both Mut and WT EGFR lung cancer cell lines 24 hours after treatment with U0126 (2 $\mu \mathrm{M})$. Gray area indicates the isotype control, and solid line indicates nontreated cells. FCM data are representative of 3 independent experiments. Data in dot plots (means $\pm \mathrm{SE}$ ) are representative of 3 independent experiments. MFI, median fluorescence intensity; iso, isotype control. To evaluate significance, ANOVA followed by Dunnett's multiple comparison test was used for $\mathbf{A}-\mathbf{C}$, or the 2 -sample $t$ test for $\mathbf{D} .{ }^{* *} P<0.01$, ${ }^{* * *} P<0.001$. (E) Representative IHC of SEMA7A and pS6 in human lung adenocarcinoma samples. Scale bar: $50 \mu \mathrm{m}$. (F) Correlation between pS6 and SEMA7A expression (IHC score) in clinical samples. Spearman's rank-order correlation was used.

inoculation (Supplemental Figure 13). Based on SEMA7A IHC, patients with scores of 0 or 1 were classified into the low-SEMA7A group, and those with scores of 2 or 3 were classified into the high-SEMA7A group. SEMA7A expression did not correlate with major clinical parameters (Table 4). We analyzed the correlation between SEMA7A expression and PFS in stage IV lung adenocarcinoma patients treated with EGFR-TKI, and we found that the low-SEMA7A group was more sensitive to EGFRTKI than the high-SEMA7A group (Figure 6E; log-rank test $P=0.0396$ ). These results indicate that SEMA7A expression predicts the response to EGFR-TKI treatment in stage IV lung adenocarcinoma patients carrying an EGFR mutation.

\section{Discussion}

In this study, we showed that the expression of SEMA7A in human lung adenocarcinoma is regulated by mTOR signaling, of which EGFR is an upstream regulator. We also demonstrated that the SEMA7AITGB1-ERK axis plays an important role in developing resistance to EGFR-TKIs by inhibiting apoptosis through activation of ERK signaling. 
Table 3. Correlations between the expression of pS6 and clinical features

\begin{tabular}{|c|c|c|c|c|c|}
\hline p56 score & $0(n=2)$ & $1(n=67)$ & $2(n=56)$ & $3(n=10)$ & significance \\
\hline Age (Mean; range) & $69.2(59-78)$ & $68.5(40-90)$ & $66.9(39-85)$ & $64.1(37-74)$ & n.s. \\
\hline Stage (Mean; range) & $3(3)$ & $2.0(1-4)$ & $2.5(1-4)$ & $3.2(1-4)$ & n.s. \\
\hline $\begin{array}{l}\text { Mutation status } \\
\text { (WT/EGFR/ALK/unknown) }\end{array}$ & $0 / 2 / 0 / 0$ & $13 / 15 / 1 / 38$ & $11 / 20 / 1 / 24$ & $3 / 2 / 1 / 4$ & n.s. \\
\hline
\end{tabular}

(Mean; range)

The Kruskal-Wallis test was used to evaluate significance.

Our transcriptome analysis revealed that Sema7A was highly induced by EGFR signaling. Driver mutation signals, including EGFR mutation, evoked diverse signaling cascades, leading not only to changes in cellular characteristics such as proliferation and survival, but also to primary and acquired drug-tolerant states. In this context, we first sought to determine which downstream signals of EGFR were involved in the expression of SEMA7A. Activated EGFR-Mut stimulates downstream oncogenic signaling pathways, including $\mathrm{PI} 3 \mathrm{~K} / \mathrm{AKT} / \mathrm{mTOR}$ and RAS/RAF/MEK/ERK. Using inhibitors of these signaling pathways, we determined that mTOR signaling positively regulates the expression of SEMA7A. Consistent with this, expression of SEMA7A and the level of pS6, as determined by IHC, were correlated in clinical samples. These results indicate that the EGFR-mTOR axis plays a key role in the induction of SEMA7A. Because mTOR is a hub for various external stimuli, expression of SEMA7A can be induced by upstream signals other than EGFR. Indeed, when we cotreated EGFR-Mut cells with an EGFR-TKI plus an mTOR inhibitor, the combination treatment resulted in higher sensitivity to EGFR-TKI than EGFR-TKI alone (Supplemental Figure 14).

From a clinical standpoint, intrinsic and acquired forms of resistance to EGFR-TKI represent critical problems to be solved. In this study, we showed that SEMA7A is involved in development of EGFR-TKI resistance after relatively prolonged treatment both in vitro and in vivo, although it is not relevant to drug resistance at the start of therapy. In this context, it is plausible that SEMA7A is prompting an enabling mechanism, subsequently leading to the development of acquired drug resistance. In addition, we found that SEMA7A promoted resistance to EGFR-TKI by activating ERK via ITGB1. Consistent with this, MEK inhibitors promoted sensitivity to EGFR-TKI in SEMA7A WT cells (Figure 6D), but such effects were absent in SEMA7A KO cells. These results support the importance of the SEMA7A-ITGB1-ERK axis in resistance mechanisms. Indeed, it is noteworthy that ERK signaling through ITGB1 is critical for the biological activities of SEMA7A in neural cells and macrophages $(15,19,20)$, suggesting that the ITGB1-ERK axis promotes resistance to EGFR-TKI $(34,35)$. Thus, it appears that SEMA7A functions as a ligand of ITGB1 and activates ERK signaling, which in turn induces resistance to EGFR-TKIs. Our investigations did not fully clarify why differences of ERK activation and EGFR-TKI sensitivity emerge later in the long exposure to EGFR-TKIs. We hypothesized that, at the baseline time point, ERK activation is probably saturated by mutated EGFR signaling; thus, the SEMA7A-ITGB1-ERK axis is dispensable. However, during longer exposure to EGFR-TKIs, ERK activation may come to depend on the SEMA7A-ITGB1 axis, eventually resulting in drug tolerance and resistance. In other words, the requirement of SEMA7A for cell survival and proliferation changes over the course of longer exposures to EGFR-TKIs. Regarding other possible resistance mechanisms mediated by SEMA7A, we observed neither T790M, MET overexpression, HGF overexpression (36), nor EMT in our cell culture experiments. These findings are summarized in Figure 7, which depicts a model in which SEMA7A and ITGB1 interact each other, either in trans or in cis. It is also possible that SEMA7A contributes to drug resistance by mediating interactions between cancer cells and components in the tumor microenvironment. Because EGFR-mutated mouse lung adenocarcinoma cell lines are not available, we cannot inoculate cancer cells into the lungs of immunocompetent mice and analyze the functions of SEMA7A as an activator of the tumor microenvironment containing macrophage and immune cells. In addition, because it is difficult to perform rebiopsy, we do not have enough clinical samples to confirm SEMA7A expression in EGFR-Mut NSCLCs that have become resistant to EGFR-TKIs. Further investigations are required to fully elucidate the role of SEMA7A in EGFR-TKI resistance. 


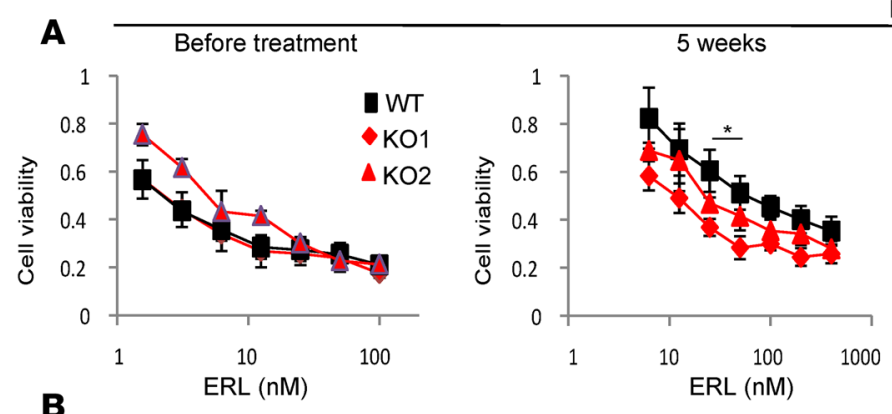

$\mathrm{HCC} 827$
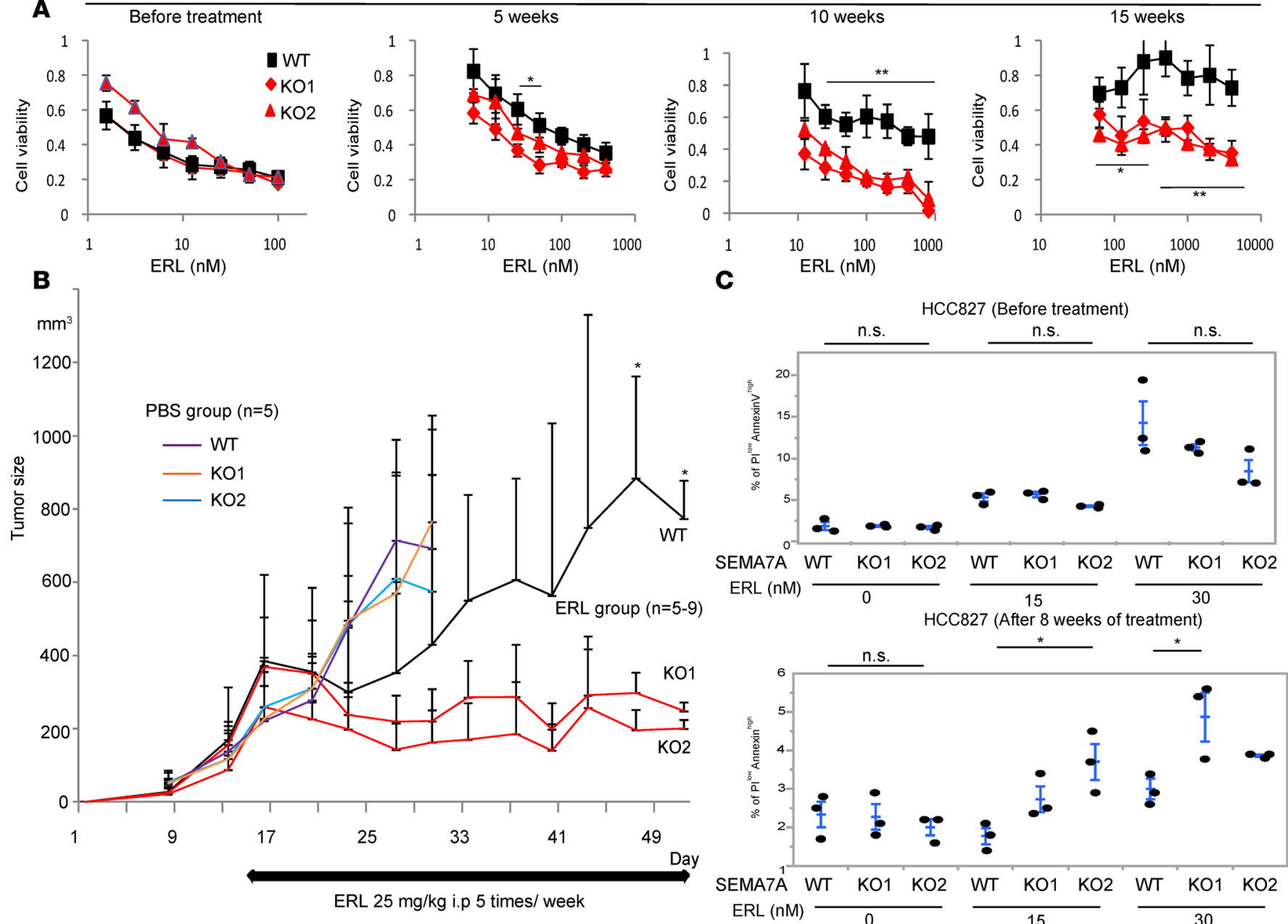

Figure 4. SEMA7A-expressing lung adenocarcinoma cells become resistant to EGFR-TKI due to antiapoptotic activity. (A) SEMA7A WT and KO HCC827 cells were treated with low-dose erlotinib for $0,5,10$, or 15 weeks. Treated cells were passaged and cultured on 96 -well plates for 24 hours and were then exposed to erlotinib at the indicated concentrations for 72 hours, followed by modified MTT assay. Data (means \pm SE) are representative of 3 independent experiments. (B) Growth of SEMA7A WT or KO HCC827 xenograft tumors. SEMA7A WT or KO HCC827 xenografted mice were treated with erlotinib (ERL; $25 \mathrm{mg} / \mathrm{kg}, 5$ times per week). The mice became emaciated after 5 weeks of ERL treatment; thus, we could not continue further observations. Data are representative of 3 independent experiments and are displayed as means \pm SE. (C) SEMA7A WT and KO HCC827 cells were exposed to erlotinib at the indicated concentrations for 48 hours, before and after treatment with low-dose erlotinib for 8 weeks. Graphs indicate the proportion of $\mathrm{Pl}^{10}$ and Annexin $\mathrm{V}^{\text {hi }}$ cell populations, expressed as percentages. The experiments were performed in triplicate, and data are displayed as means \pm SE. To evaluate significance, ANOVA followed by Dunnett's multiple comparison test was used. ${ }^{*} P<0.05$, ${ }^{*} P<0.01$.

Among patients with EGFR-Mut, some respond well to EGFR-TKIs, whereas others do not. Currently, no established method is available for distinguishing good and poor responders prior to treatment. In this study, we demonstrated that SEMA7A is a potentially useful predictive biomarker in EGFRMut lung adenocarcinoma patients. In addition, SEMA7A is a candidate therapeutic target. Although EGFR-TKIs downregulate SEMA7A expression, it is worthy of note that we still observed residual SEMA7A expression (Supplemental Figure 6), which was crucially involved in drug resistance (Figure 4A). Combination therapy with EGFR-TKIs and inhibitors of the SEMA7A-ITGB1-ERK axis, such as MEK inhibitors and anti-SEMA7A neutralizing antibodies, is a promising approach (Figure 6D). SEMA7A is detected in brain, lung, sexual organs, skin, and immune cells (information from The Human Protein Atlas; https://www.proteinatlas.org). Although SEMA7A KO mice are viable and fertile, they exhibit abnormalities in inflammatory responses (19). These observations should be kept in mind when considering clinical applications, such as anti-SEMA7A neutralizing antibodies, although such antibodies are not available currently. Alternatively, combination therapy using EGFR-TKI and MEK inhibitors represents a promising strategy for conquering EGFR-TKI resistance. Indeed a MEK 
A MET/ ACTB

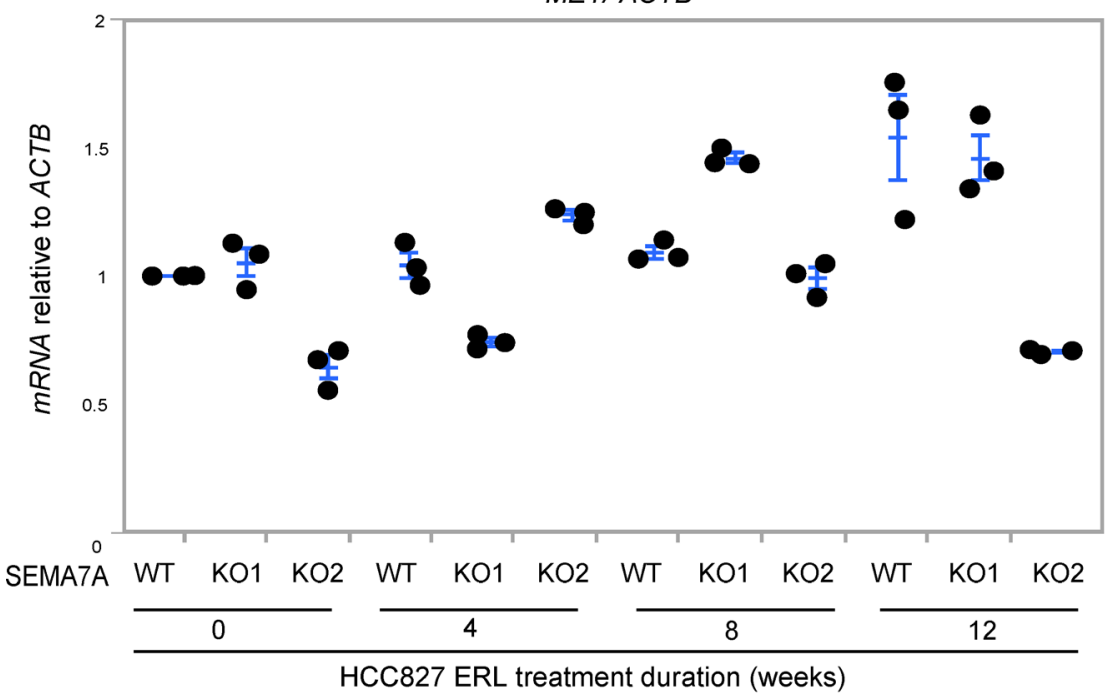

B

HCC827 (Before treatment)

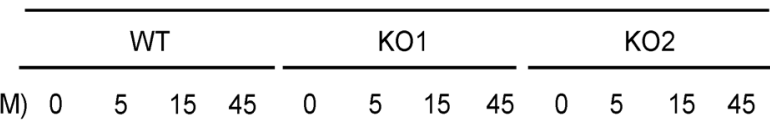

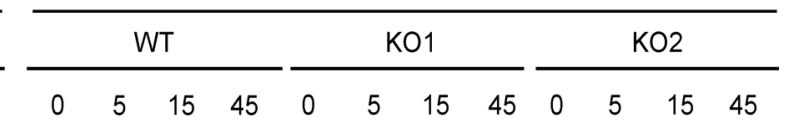

$\begin{array}{lllllllllllll}\mathrm{ERL}(\mathrm{nM}) & 0 & 5 & 15 & 45 & 0 & 5 & 15 & 45 & 0 & 5 & 15 & 45 \\ & & & & & & & & & & & & \end{array}$
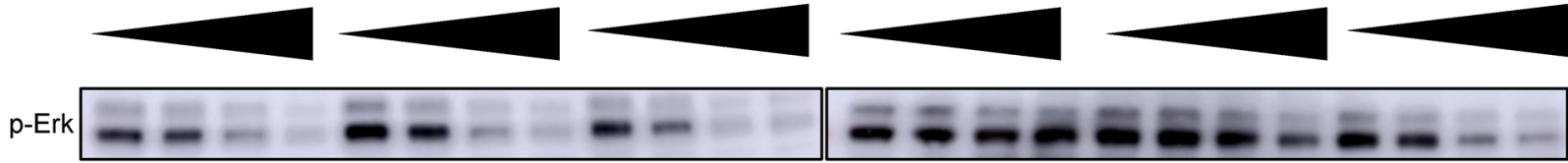

Erk
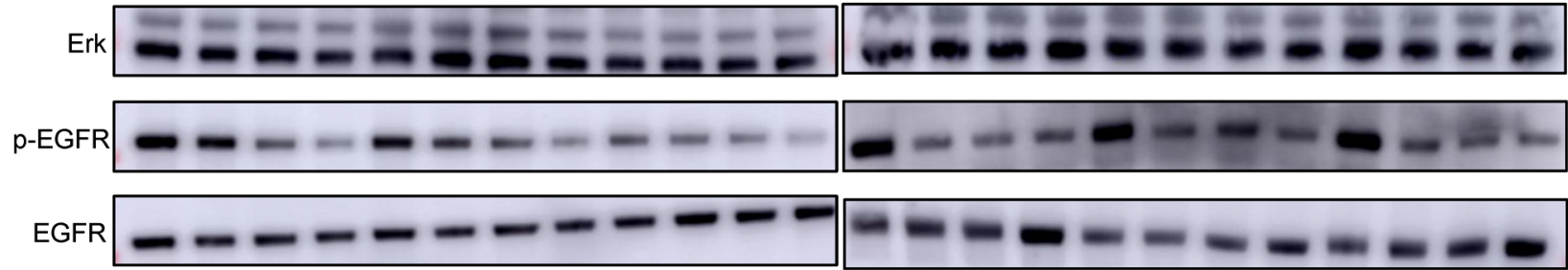

$\mathrm{ACTB}$
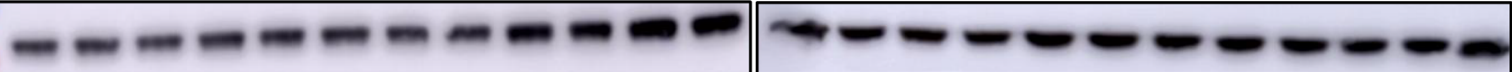

Figure 5. Erk phosphorylation is upregulated in SEMA7A WT cells without common resistant mechanisms. (A) Representative qPCR of MET in SEMA7A WT and KO HCC827 cells after low-dose exposure to erlotinib. Data (means \pm SE) are representative of 3 independent experiments. (B) Representative immunoblots showing $p$-EGFR and p-Erk in HCC827 cells 24 hours after treatment with erlotinib. After long-term treatment, the $p$-Erk level was maintained in SEMA7A WT cells, despite a reduction in the level of $p$-EGFR.

inhibitor, trametinib, has been approved for use in lung cancer patients with BRAF mutation (37), which will allow us to conduct clinical trials more safely and reasonably.

No previous studies have shown that SEMA7A inhibits the effect of anticancer drugs, and this is the first report to our knowledge demonstrating a role for SEMA7A in EGFR-TKI resistance. In conclusion, the SEMA7A-ITGB1-ERK axis is a potentially novel biomarker for predicting EGFR-TKI resistance, as well as a therapeutic target in patients with EGFR-Mut lung adenocarcinoma. Further studies are required to validate its significance and importance in lung cancer and other organ malignancies.

\section{Methods}

Cell lines and cell culture. HCC827 was a gift from Bruce E Johnson. NIH3T3, H3255, H1975, H441, H1792, and H292 cells were purchased from the American Type Culture Collection. Plat GP cells were purchased from CosmoBio. II-18 was a gift from the Cell Resource Center for Biomedical Research, Institute of Development, Aging, and Cancer, Tohoku University, Sendai, Japan. The biological properties and origin of SCLC 
Table 4. Correlations between the expression of SEMA7A and clinical features in stage IV EGFR-Mut lung adenocarcinoma

\begin{tabular}{lccc}
\hline SEMA7A score & $\begin{array}{c}\text { Low (score } \mathbf{0} \text { or } \mathbf{1}) \\
(\boldsymbol{n}=\mathbf{2 0})\end{array}$ & $\begin{array}{c}\text { High (score 2 or 3) } \\
(\boldsymbol{n}=\mathbf{2 0})\end{array}$ & Significance \\
Sex (male/female) & $6 / 14$ & $10 / 10$ & n.s. \\
Age (Mean; range) & $71.7(39-81)$ & $65.4(52-84)$ & n.s. \\
Brinkman index (Mean; range) & $137(0-680)$ & $248(0-2000)$ & n.s. \\
Performance status (Mean; range) & $0.70(0-2)$ & $0.75(0-2)$ & n.s. \\
First-line EGFR-TKI & $10 / 6 / 4$ & $12 / 6 / 2$ & n.s. \\
(Gefitinib/erlotinib/afatinib) & & & \\
\hline
\end{tabular}

The Kruskal-Wallis test was used to evaluate significance.

cell lines, including H69, SBC-3, and CADO LC6, were described previously $(38,39)$. EGFR genotypes of the adenocarcinoma cell lines are as follows: HCC827, del19; H3255, L858R; II-18, L858R; H1975, L858R/ T790M; and H441 and H292, WT. NIH3T3 cells were cultured in DMEM (Nacalai Tesque) supplemented with 10\% heat-inactivated bovine serum (Thermo Fisher Scientific), $100 \mathrm{U} / \mathrm{ml}$ penicillin, and $100 \mathrm{mg} / \mathrm{ml}$ streptomycin (Nacalai Tesque). Other cells were cultured in RPMI-1640 (Nacalai Tesque) supplemented with $10 \%$ heat-inactivated FBS (MilliporeSigma), $100 \mathrm{U} / \mathrm{ml}$ penicillin, and $100 \mathrm{mg} / \mathrm{ml}$ streptomycin.

EGFR- or SEMA7A-expressing cells. WT and Mut EGFR (exon 19 deletion) were constructed as previously reported (40). Human SEMA7A expression vectors were purchased from Open Biosystems (GE Dharmacon). All vectors were subcloned into MSCV retroviral vectors (Clontech). These vectors were cotransfected with pVSV-G (Riken) into Plat-GP packaging cells using the Lipofectamine 2000 DNA Transfection Reagent (Thermo Fisher Scientific), and pantropic retroviral vectors were produced. WT or Mut EGFR vectors were transduced into NIH3T3 cells, and SEMA7A vectors were transduced into human lung adenocarcinoma cells. Clones stably expressing WT or Mut EGFR and SEMA7A were established by puromycin selection ( $2 \mu \mathrm{g} / \mathrm{ml}$; MilliporeSigma) for 4 days.

Transcriptome analysis. Total RNA expression profiling was performed by MBL Co. Ltd. The protocol outline was as follows: RNA was isolated and purified from WT and Mut EGFR-expressing NIH3T3 cells using the AllPrep DNA/RNA Mini kit (Qiagen). RNA was reverse-transcribed into T7-cDNA, and Cy3cRNA was obtained from an in vitro transcription reaction. The Cy3-cRNA was hybridized to the microarrays (SurePrint G3 Mouse Gene Expression 8x60K, Agilent Single-Color 28005). Raw data were imported into GeneSpring, and expression values were normalized. All entities were measured and filtered based on Flags (detected, not detected, and compromised) and selected by reproducibility of spot signals. Fold change (FC) was calculated for upregulated genes by dividing EGFR-Mut values by WT EGFR values.

KO of SEMA7A. Cells at 70\%-90\% confluence were transfected using Lipofectamine 2000 DNA Transfection Reagent. HCC827 or H3255 cells were seeded in 60-mm plates, and cotransfection was performed using guide RNA (gRNA) vectors ( $2 \mu \mathrm{g}$ plasmid per well) and the Hygromycin linear marker (Clontech). The gRNA vectors were custom-designed CRISPR-Cas9 vectors (px330-U6-Chimeric_BB-CB-hSpCas9; obtained from Addgene, catalog 42230) (41), targeting 2 specific regions of human SEMA7A (Supplemental Figure 4A). Hygromycin $(100 \mu \mathrm{g} / \mathrm{ml})$ selection was started 48 hours after transfection. After clonal expansion, genomic DNA was obtained using the AllPrep DNA/RNA Mini kit (Qiagen). The 2 target regions were amplified by PCR using forward and reverse primers upstream and downstream of the expected mutation sites (Supplemental Figure 4D). PCR conditions were as follows: denaturation at $94^{\circ} \mathrm{C}$ for 5 minutes; 30 cycles of $94^{\circ} \mathrm{C}$ for 30 seconds, $59^{\circ} \mathrm{C}$ for 60 seconds, and $72^{\circ} \mathrm{C}$ for 60 seconds; and a final extension cycle of $72^{\circ} \mathrm{C}$ for 7 minutes. The cycle sequencing reaction was run on an ABI2700 instrument (Thermo Fisher Scientific). The PCR products were sequenced by the Sanger method at The Center for Medical Research and Education, Osaka University.

Knockdown of ITGB1. Small hairpin (sh) RNAs were provided by the Center for Medical Research and Education, Graduate School of Medicine, Osaka University. The shRNA plasmids targeting the human ITGB1 gene (TRCN0000029645, TRCN0000029646, and TRCN0000029648) were derived from the MISSION TRC-Hs1.0 system. The negative control vector, MISSION pLKO.1-puro Non-Target shRNA Control Plasmid DNA, was purchased from MilliporeSigma.

Antibodies and drugs. Antibodies for Western blots were purchased from indicated suppliers: $\beta$-actin 
A

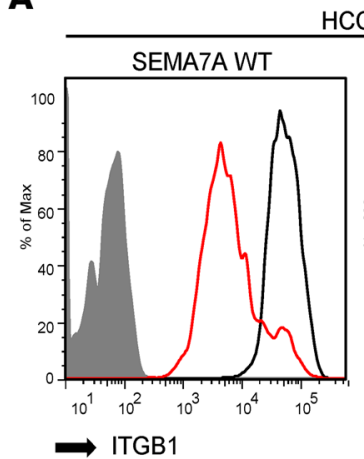

$\mathrm{HCC} 827$

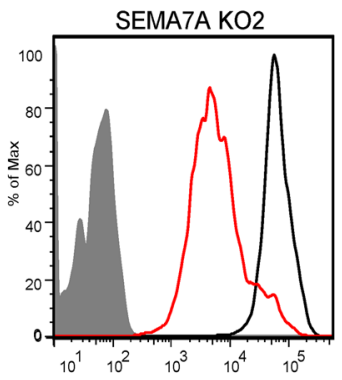

$\rightarrow$ ITGB

C
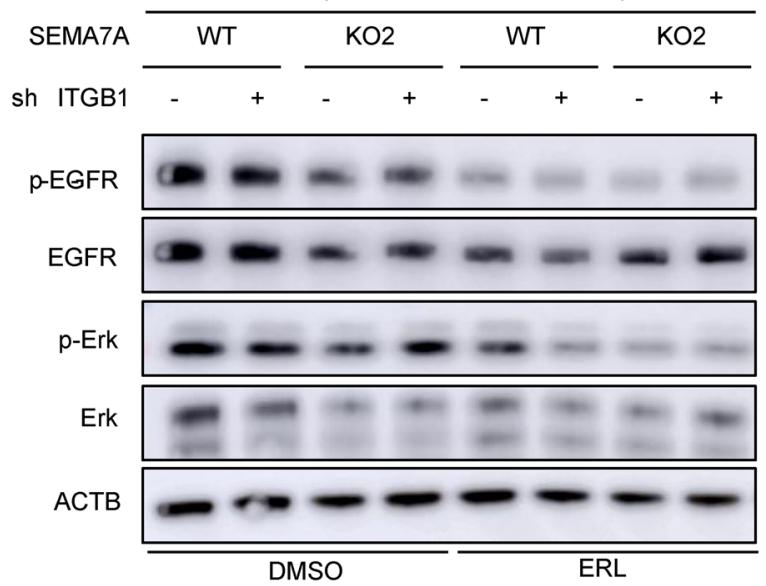

E

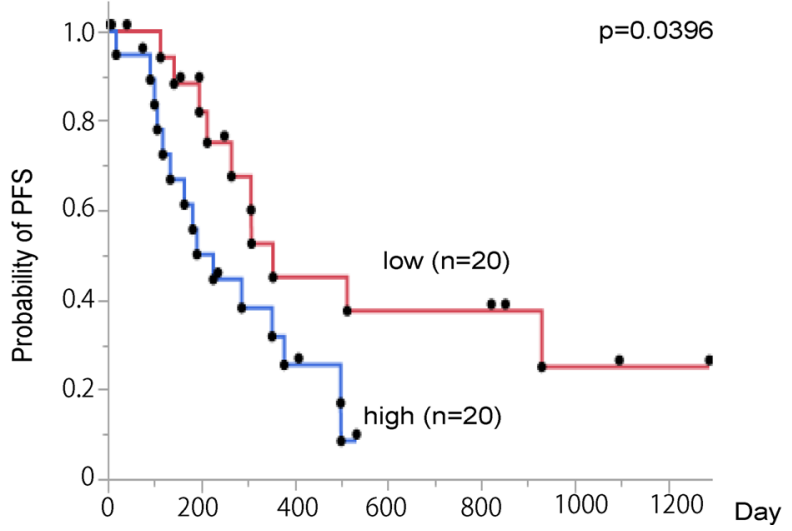

Isotype ctrl

sh Ctrl

ITGB1

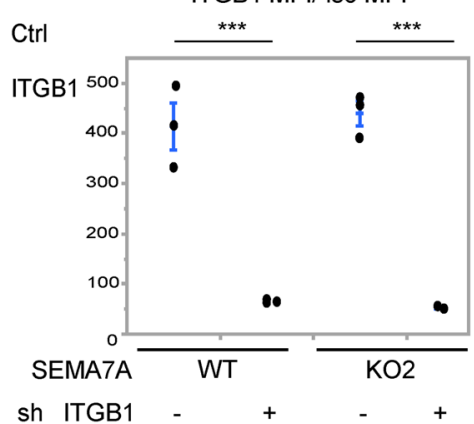

sh ITGB1
ITGB1 MFI/ iso MFI

HCC827
B

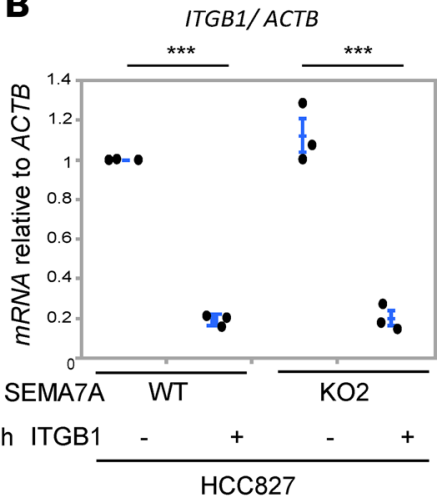

D

D $\mathrm{HCC} 827$

(After 6 weeks of treatment)

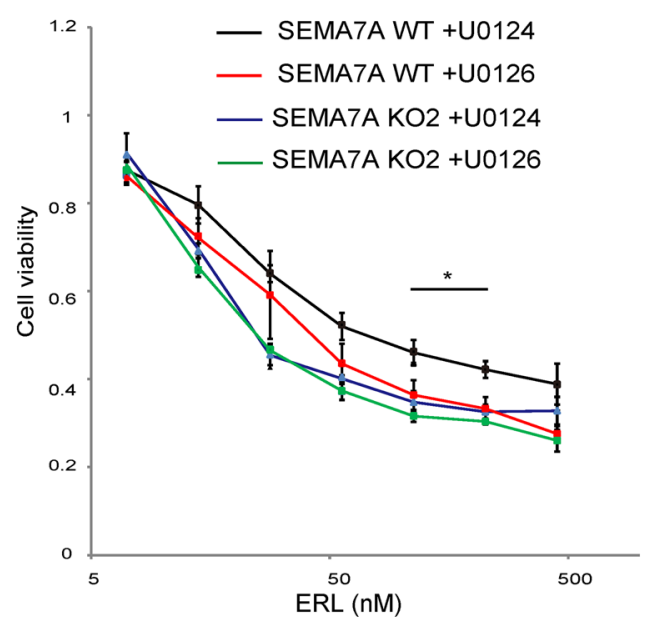

Figure 6. The SEMA7A-ITCB1 axis regulates Erk phosphorylation under erlotinib treatment. (A and B) ITCB1 expression in SEMA7A WT or KO HCC827 cells generated by sh-Ctrl or sh-ITCB1 RNA, tested by FCM and qPCR. Data (means \pm SE) are representative of 3 independent experiments. $\mathrm{MFI}$, median fluorescence intensity; iso, isotype control. Gray area indicates the isotype control. The 2-sample $t$ test was used to detect significance. ${ }^{* *} P<0.001$. (C) Representative immunoblots showing p-EGFR and p-Erk in HCC827 cells 24 hours after treatment with erlotinib. After long-term treatment, the $p$-Erk level was maintained in SEMA7A WT cells, despite a reduction in the level of p-EGFR, which was suppressed by knockdown of ITCB1. (D) SEMA7A WT and KO HCC827 cells were treated with low-dose erlotinib for several weeks. Treated cells were passaged and cultured on 96-well plates for 24 hours, exposed to erlotinib and U0124 $(5 \mu \mathrm{M})$ or U0126 $(5 \mu \mathrm{M})$ for 72 hours, and then subjected to modified MTT assay. Data (means \pm SE) are representative of 3 independent experiments. To evaluate significance, ANOVA followed by Tukey-Kramer multiple comparison test was used. ${ }^{*} P<0.05$. (E) PFS for patients with EGFR mutation for whom EGFR-TKI was used as the first-line treatment. The $P$ value for the difference between the 2 curves was determined by the log-rank test. IHC scores were defined as follows: 0, 0\%-1\% positive tumor cells; $1,1 \%-20 \% ; 2,21 \%-50 \% ; 3,51 \%-100 \%$. Based on SEMA7A IHC, patients with scores of 0 or 1 were classified into the low-SEMA7A group, and those with scores of 2 or 3 were classified into the high-SEMA7A group. 


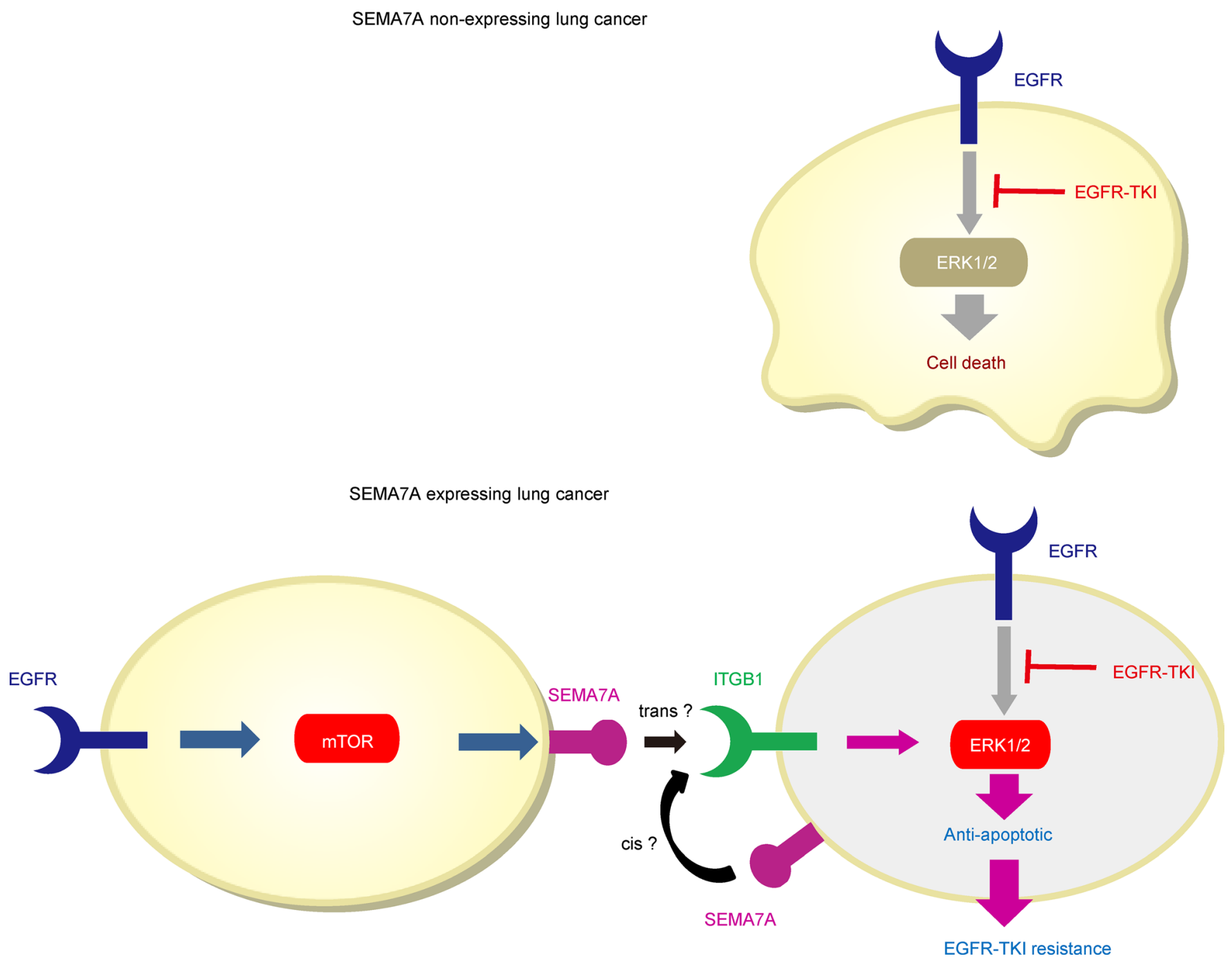

Figure 7. Graphical summary of this study. Expression of SEMA7A in human lung adenocarcinoma is regulated by the EGFR-mTOR axis and is involved in development of resistance to EGFR-TKIs. Resistance is mediated by inhibition of apoptosis by the SEMA7A-ITCB1-ERK axis.

(catalog 4970), p-EGFR (Tyr1068, catalog 2234), S6K (catalog 2708), p-S6K (T389, catalog 9234), Akt (catalog 4691), p-Akt (Ser473, catalog 4060), Erk1/2 (catalog 9102), and p-Erk1/2 (Thr202/Tyr204, catalog 9101) from Cell Signaling Technology; and EGFR (catalog sc-03) from Santa Cruz Biotechnology.

Horseradish peroxidase-conjugated secondary antibodies were purchased from GE Healthcare. Antibodies and reagents for FCM were purchased from the indicated suppliers: nonconjugated antimouse SEMA7A (clone SKK-7, Abnova) biotinylated with Biotin Labeling Kit-NH2 (DOJINDO), biotin-conjugate anti-mouse IgG1 $\kappa$ isotype control (clone P3.6.2.8.1, eBioscience), PE-conjugate antihuman SEMA7A (clone MEM-150, BioLegend), PE-conjugated mouse IgM isotype control (clone MM-30; BioLegend), APC-conjugated anti-human ITGB1 (clone TS2/16, BioLegend), APC-conjugated mouse IgG1 $\kappa$ isotype control (clone MOPC-21, BioLegend), FITC-conjugated CD45 antibody (clone HI30, BioLegend), APC-conjugated EGFR (clone AY13, BioLegend), and 7-AAD (BioLegend). Antibodies for IHC were purchased from the indicated suppliers: SEMA7A (catalog sc-135263, Santa Cruz Biotechnology Inc.), phospho-S6 ribosomal protein (clone D57.2.2E, Cell Signaling Technology), and ITGB1 (clone EP1041Y, MilliporeSigma).

DMSO was purchased from WAKO. Carboplatin was purchased from MilliporeSigma. Erlotinib was purchased from Chemietek for animal experiments and Selleck Chemicals for cell culture experiments. Osimertinib was purchased from Selleck Chemicals. Recombinant human EGF was purchased from Thermo Fisher Scientific. U0124 and U0126 were purchased from Calbiochem. 
Western blots. Protein samples were loaded onto NuPAGE 4-12\% Bis-Tris gels (Invitrogen). For immunoblot analysis, the gel was electroblotted onto a polyvinylidene difluoride membrane (Bio-Rad). The membrane was blocked with Blocking One (Nacalai Tesque) or Blocking One P (Nacalai Tesque), incubated with specific primary antibody, and then incubated with the appropriate secondary antibody. Signals were detected on an ImageQuant LAS500 (GE Healthcare).

FCM. Cells were stained for FCM analysis of cell surface markers with the following fluorochrome-conjugated Abs diluted in PBS (Nacalai Tesque) containing 2\% FBS. Cells were stained with primary antibody for 15 minutes on ice. After staining of cell-surface molecules, SEMA7A expression levels were determined for the 7-AAD-negative (1:200) population.

Cells from malignant pleural effusions were blocked with Fc-block (1:10; Miltenyi Biotec) for 15 minutes at room temperature (RT) and were then stained for 20 minutes at RT with FITC-conjugated CD45 antibody and PE-conjugated SEMA7A antibody or isotype control. Red cells were destroyed by incubation with RBC Lysis Buffer (eBioscience) for 15 minutes at RT.

The SEMA7A WT or KO xenografts were obtained 2 weeks after administration of erlotinib and shredded into small pieces, followed by incubation in collagenase-containing buffer: $100 \mathrm{U} / \mathrm{ml}$ of collagenase type IV (Invitrogen), $50 \mathrm{~g} / \mathrm{ml}$ of DNase I (Roche Diagnostics), and 10\% FBS in RPMI-1640 medium for 45 minutes. After incubation, cells were passed through a cell strainer to remove debris. The cell pellet was dissolved in 2\% FBS, blocked with Fc-block (1:10; Miltenyi Biotec) for 15 minutes on ice, and then stained with APC-conjugated EGFR antibody and PE-conjugated SEMA7A antibody or isotype control for 20 minutes at RT.

Stained cells were analyzed on a BD FACS Canto II flow cytometer equipped with the Diva software (BD Biosciences). Final analysis and graphical output were performed using FlowJo (TreeStar).

$q R C R$. Total RNA was extracted using the RNeasy Mini Kit (Qiagen), and cDNA was synthesized using the Super Script III cDNA synthesis kit (Invitrogen). qPCR analysis was performed on a 7900HT Fast Real-Time PCR system (Applied Biosystems) using TaqMan PCR protocols. TaqMan real-time probes for human SEMA7A/CD108 (Hs01118882_m1), human ACTB (Hs01060665_g1), human MET (Hs01565584_m1), human ITGB1 (Hs00559595_m1), and mouse Sema7a/Cd108 (Mm00441361_m1) were purchased from Applied Biosystems. Samples were run in triplicate using $A C T B$ or Gapdh as an internal control, and the $\Delta \Delta \mathrm{CT}$ method was used for relative mRNA calculations.

Establishment of an erlotinib-or osimertinib-resistant subpopulation of HCC827, H3255, and II-18 cells. To establish resistant subpopulations, HCC827 and H3255 cells were treated with $2.5 \mathrm{nM}$ erlotinib, and II-18 cells were treated with $10 \mathrm{nM}$ erlotinib. HCC 827 cells were also treated with $2.5 \mathrm{nM}$ osimertinib. Each drug concentration was lower than the $\mathrm{IC}_{50}$ in each cell line. Subsequently, the concentration was increased in a stepwise manner.

Modified MTT assay. Growth inhibition was measured by a modified 3-(4,5-dimethylthiazol-2-yl)-2,5diphenyltetrazolium bromide (MTT) assay (Cell Counting Kit-8; Dojindo). Briefly, the cells were plated on 96-well plates, incubated for 1 day, and then exposed to erlotinib. Cell viability was assayed after 72 hours of incubation. Each assay was performed in quadruplicate, and means and SD were calculated.

Flow cytometric Annexin V-APC-PI apoptosis analysis. Apoptosis was compared between erlotinib-treated and nontreated HCC827 or H3255 cells using a flow-cytometric Annexin V-APC-PI apoptosis assay. Briefly, harvested cells were suspended in $100 \mu 1$ of $1 \times$ binding buffer and stained with $5 \mu 1$ each of Annexin V-APC and propidium iodide (PI; MilliporeSigma). After 15 minutes of incubation, the cells were mixed with $400 \mu 1$ of $1 \times$ binding buffer and analyzed on a BD FACS Canto II flow cytometer equipped with the Diva software. Final analysis and graphical output were performed using FlowJo.

IHC. This study was approved by the Ethics Committee of Osaka University Hospital. Written informed consent was obtained from all patients. A total of 190 patients with human lung adenocarcinoma treated at Osaka University Hospital between 2010 and 2017 were included in this study (Table 2). All specimens were fixed in $10 \%$ buffered formalin (Muto Pure Chemicals) and embedded in paraffin according to standard procedures. The sections were deparaffinized in xylene and rehydrated through graded alcohol and deionized water. Antigen retrieval was performed by autoclaving in Target Retrieval solution, $\mathrm{pH} 6$ (Dako) at $125^{\circ} \mathrm{C}$ for 15 minutes. The slides were treated with peroxidase blocking solution (Dako) at RT for 5 minutes to block nonspecific binding sites. The slides were immunostained at $4^{\circ} \mathrm{C}$ overnight with anti-SEMA7A (1:50), anti-pS6 (1:200), or anti-ITGB1 (1:50), followed by incubation with secondary anti-rabbit antibodies at RT for 1 hour. The secondary antibodies were EnVisionTM1/HRP (Dako), and then developed with 3,30-diaminobenzidine as the chromogen (Dako) at RT for 5 minutes. Two oncologists blinded to the clinical information of these 
patients independently assessed the expression of SEMA7A and pS6. All histological images were acquired on a BZ-X710 microscope (Keyence). Thirty-two patients were excluded because the samples were too small to analyze expression of SEMA7A. Scores were defined as follows: 0, $0 \%-1 \%$ positive tumor cells; $1,1 \%-20 \%$; $2,21 \%-50 \% ; 3,51 \%-100 \%$. Among patients with stage IV EGFR-Mut adenocarcinoma treated at Osaka University Hospital and Osaka International Cancer Institute between 2010 and 2017, a total of 40 cases for which both clinical data and samples were available were included in this study (Table 4). PFS was defined as the time from initiation of EGFR-TKI or chemotherapy to recurrence, death, cessation of treatment by adverse events, or last follow-up. PFS was estimated by the Kaplan-Meier method, and multivariable analyses were performed to assess survival differences.

Animal experiments. Six-week-old male BALB/cAJcl $n u / n u$ mice were obtained from CLEA Japan. HCC 827 cells $\left(3 \times 10^{6}\right)$ suspended in PBS were inoculated s.c. into the flanks of the mice. Starting 2 weeks after injection, $25 \mathrm{mg} / \mathrm{kg}$ erlotinib was administered 5 times a week i.p. Tumor volume was determined by computing width ${ }^{2} \times$ length $/ 2$.

Determination of EGFR mutations. EGFR mutation status of biopsy samples at primary diagnosis and EGFR-Mut cells in culture were determined by the PNA-LNA PCR clamp method. Rebiopsy samples to detect secondary EGFR mutation T790M were processed and analyzed using a real-time PCR-based method, the Cobas EGFR Mutation Detection kit. Both analyses were performed in the central laboratory at LSI Medicine Corporation.

Mycoplasma testing. Genome DNA from all cell lines was obtained using the AllPrep DNA/RNA Mini kit (Qiagen). Mycoplasma detection was performed using the e-Myco plus Mycoplasma PCR Detection Kit (iNtRON), and only mycoplasma-negative cells were used in this study.

Statistics. Statistical evaluations for experiments using cell lines and mice were repeated at least 3 times. Data are expressed as means \pm SE. In FCM, qPCR, and animal experiments, differences were evaluated using the 2-tailed Student's $t$ test for single between-group comparisons, while 1-way ANOVA followed by Dunnett's or Tukey-Kramer test was used for multiple comparisons. In clinical studies, nonparametric Spearman's rank-order correlation was used to compare 2 variables for Figures $2 \mathrm{~F}$ and Figure 3F. To analyze clinical parameters for the tables, the Fisher's exact test was used for comparing 2 categorical variables, and Kruskal-Wallis test followed by Steel-Dwass test was used for continuous variables. Progression free survival (PFS) was estimated using the Kaplan-Meier method, and the $P$ value for the difference between the 2 curves was determined by the log-rank test. $P$ values less than or equal to 0.05 were considered significant. All statistical analyses were performed using the JMP Pro 14.0.0 software.

Study approval. This study was approved by the ethical review board of Osaka University Hospital (approval no. 16067), Suita, Japan, in accordance with the Declaration of Helsinki. All human samples were obtained after informed consent provided by the subjects. Animal studies were approved by the ethical review board of the Osaka University Graduate School of Medicine (approval nos. 27-039-003, 28-031004; Suita, Japan), and protocols for animal experiments were approved by the Institute of Experimental Animal Sciences of Osaka University Medical School.

\section{Author contributions}

Conception and design were contributed by YK, IN, SK, TK, and AK. Development of methodology was contributed by YK, IN, SK, DI, SN, RK, NY, YS, YF, YK, MN, KM, SK, HT, and AK. Acquisition of data (e.g., provided animals, acquired and managed patients, provided facilities, ) was contributed by YK, IN, SK, SN, YS, FY, AO, TN, YH, MH, OM, TM, HH, KI, TT, YT, SH, YS, MO, TK, KN, FI, SN, TK, HK, and AK. Analysis and interpretation of data (e.g., statistical analysis, biostatistics, computational analysis) were contributed by YK, IN, and MH. Writing, review, and/or revision of the manuscript were contributed by YK, IN, NH, and AK. Administrative, technical, or material support (e.g., reporting or organizing data, constructing databases) was contributed by $\mathrm{YK}, \mathrm{IN}, \mathrm{MH}$, and $\mathrm{AK}$. Revision of article was contributed by $\mathrm{AK}$.

\section{Acknowledgments}

This research was supported by the AMED-CREST and COI stream from the Japan Agency for Medical Research and Development (AMED). The authors thank Medical and Biological Laboratories Co. Ltd. (Leave a Nest grant). This study was supported by Yuri Terao and Center for Medical Research and Education, Graduate School of Medicine, Osaka University. 
Address correspondence to: Atsushi Kumanogoh or Izumi Nagatomo, Department of Respiratory Medicine and Clinical Immunology, Graduate School of Medicine, Osaka University, 2-2 Yamada-oka, Suita, Osaka 565-0871, Japan. Phone: 81.6.6879.3830; Email: kumanogo@imed3.med.osaka-u.ac.jp (A. Kumanogoh). Phone: 81.6.6879.3833; Email: iznagatomo@imed3.med.osaka-u.ac.jp (I. Nagatomo).

1. Jemal A, et al. Cancer statistics, 2008. CA Cancer J Clin. 2008;58(2):71-96.

2. Ettinger DS, et al. Non-small cell lung cancer clinical practice guidelines in oncology. J Natl Compr Canc Netw. 2006;4(6):548-582.

3. International Early Lung Cancer Action Program Investigators, et al. Survival of patients with stage I lung cancer detected on CT screening. N Engl J Med. 2006;355(17):1763-1771.

4. Fukuoka M, et al. Biomarker analyses and final overall survival results from a phase III, randomized, open-label, first-line study of gefitinib versus carboplatin/paclitaxel in clinically selected patients with advanced non-small-cell lung cancer in Asia (IPASS). J Clin Oncol. 2011;29(21):2866-2874.

5. Paez JG, et al. EGFR mutations in lung cancer: correlation with clinical response to gefitinib therapy. Science. 2004;304(5676):1497-1500.

6. Lynch TJ, et al. Activating mutations in the epidermal growth factor receptor underlying responsiveness of non-small-cell lung cancer to gefitinib. N Engl J Med. 2004;350(21):2129-2139.

7. Mok TS, et al. Gefitinib or carboplatin-paclitaxel in pulmonary adenocarcinoma. N Engl J Med. 2009;361(10):947-957.

8. Pao W, et al. Acquired resistance of lung adenocarcinomas to gefitinib or erlotinib is associated with a second mutation in the EGFR kinase domain. PLoS Med. 2005;2(3):e73.

9. Kobayashi S, et al. EGFR mutation and resistance of non-small-cell lung cancer to gefitinib. N Engl J Med. 2005;352(8):786-792.

10. Bean J, et al. MET amplification occurs with or without T790M mutations in EGFR mutant lung tumors with acquired resistance to gefitinib or erlotinib. Proc Natl Acad Sci USA. 2007;104(52):20932-20937.

11. Engelman JA, et al. MET amplification leads to gefitinib resistance in lung cancer by activating ERBB3 signaling. Science. 2007;316(5827):1039-1043.

12. Maemondo M, et al. Gefitinib or chemotherapy for non-small-cell lung cancer with mutated EGFR. $N$ Engl J Med. 2010;362(25):2380-2388.

13. Mitsudomi T, et al. Gefitinib versus cisplatin plus docetaxel in patients with non-small-cell lung cancer harbouring mutations of the epidermal growth factor receptor (WJTOG3405): an open label, randomised phase 3 trial. Lancet Oncol. 2010;11(2):121-128.

14. Suda K, Bunn PA, Rivard CJ, Mitsudomi T, Hirsch FR. Primary Double-Strike Therapy for Cancers to Overcome EGFR Kinase Inhibitor Resistance: Proposal from the Bench. J Thorac Oncol. 2017;12(1):27-35.

15. Pasterkamp RJ, Peschon JJ, Spriggs MK, Kolodkin AL. Semaphorin 7A promotes axon outgrowth through integrins and MAPKs. Nature. 2003;424(6947):398-405.

16. Czopik AK, Bynoe MS, Palm N, Raine CS, Medzhitov R. Semaphorin 7A is a negative regulator of T cell responses. Immunity. 2006;24(5):591-600

17. Suzuki K, Kumanogoh A, Kikutani H. Semaphorins and their receptors in immune cell interactions. Nat Immunol. 2008;9(1):17-23.

18. Miyazaki N, et al. Developmental localization of semaphorin H messenger RNA acting as a collapsing factor on sensory axons in the mouse brain. Neuroscience. 1999;93(1):401-408

19. Suzuki K, et al. Semaphorin 7A initiates T-cell-mediated inflammatory responses through alpha1beta1 integrin. Nature. 2007;446(7136):680-684.

20. Kang S, et al. Intestinal epithelial cell-derived semaphorin 7A negatively regulates development of colitis via $\alpha v \beta 1$ integrin J Immunol. 2012;188(3):1108-1116

21. Ringnér M, Fredlund E, Häkkinen J, Borg Å, Staaf J. GOBO: gene expression-based outcome for breast cancer online. PLoS One. 2011;6(3):e17911

22. Allegra M, et al. Semaphorin-7a reverses the ERF-induced inhibition of EMT in Ras-dependent mouse mammary epithelial cells. Mol Biol Cell. 2012;23(19):3873-3881.

23. Garcia-Areas R, et al. Semaphorin7A promotes tumor growth and exerts a pro-angiogenic effect in macrophages of mammary tumor-bearing mice. Front Physiol. 2014;5:17.

24. Black SA, Nelson AC, Gurule NJ, Futscher BW, Lyons TR. Semaphorin 7a exerts pleiotropic effects to promote breast tumor progression. Oncogene. 2016;35(39):5170-5178.

25. Saito T, et al. Semaphorin7A Promotion of Tumoral Growth and Metastasis in Human Oral Cancer by Regulation of G1 Cell Cycle and Matrix Metalloproteases: Possible Contribution to Tumoral Angiogenesis. PLoS One. 2015;10(9):e0137923.

26. Ma B, et al. Role of chitinase 3-like-1 and semaphorin 7a in pulmonary melanoma metastasis. Cancer Res. 2015;75(3):487-496.

27. Formolo CA, Williams R, Gordish-Dressman H, MacDonald TJ, Lee NH, Hathout Y. Secretome signature of invasive glioblastoma multiforme. J Proteome Res. 2011;10(7):3149-3159.

28. Lazova R, Gould Rothberg BE, Rimm D, Scott G. The semaphorin 7A receptor Plexin C1 is lost during melanoma metastasis Am J Dermatopathol. 2009;31(2):177-181.

29. Scott GA, McClelland LA, Fricke AF, Fender A. Plexin C1, a receptor for semaphorin 7a, inactivates cofilin and is a potential tumor suppressor for melanoma progression. J Invest Dermatol. 2009;129(4):954-963.

30. Guinot A, Oeztuerk-Winder F, Ventura JJ. miR-17-92/p38 $\alpha$ Dysregulation Enhances Wnt Signaling and Selects Lgr6+ Cancer Stem-like Cells during Lung Adenocarcinoma Progression. Cancer Res. 2016;76(13):4012-4022.

31. Geng R, et al. RNF183 promotes proliferation and metastasis of colorectal cancer cells via activation of NF-kB-IL-8 axis. Cell Death Dis. 2017;8(8):e2994.

32. Haber DA, et al. Molecular targeted therapy of lung cancer: EGFR mutations and response to EGFR inhibitors. Cold Spring Harb Symp Quant Biol. 2005;70:419-426. 
33. Camp ER, Summy J, Bauer TW, Liu W, Gallick GE, Ellis LM. Molecular mechanisms of resistance to therapies targeting the epidermal growth factor receptor. Clin Cancer Res. 2005;11(1):397-405.

34. Li ZX, et al. Mig-6 overcomes gefitinib resistance by inhibiting EGFR/ERK pathway in non-small cell lung cancer cell lines. Int J Clin Exp Pathol. 2014;7(10):7304-7311.

35. Ochi N, et al. Src mediates ERK reactivation in gefitinib resistance in non-small cell lung cancer. Exp Cell Res. 2014;322(1):168-177.

36. Yano S, et al. Hepatocyte growth factor induces gefitinib resistance of lung adenocarcinoma with epidermal growth factor receptor-activating mutations. Cancer Res. 2008;68(22):9479-9487.

37. Planchard D, et al. Dabrafenib plus trametinib in patients with previously untreated BRAFV600E-mutant metastatic non-smallcell lung cancer: an open-label, phase 2 trial. Lancet Oncol. 2017;18(10):1307-1316.

38. Mitsuhashi Y, Inaba M, Sugiyama Y, Kobayashi T. In vitro measurement of chemosensitivity of human small cell lung and gastric cancer cell lines toward cell cycle phase-nonspecific agents under the clinically equivalent area under the curve. Cancer. 1992; 70(10):2540-2546.

39. Minami T, et al. HER2 as therapeutic target for overcoming ATP-binding cassette transporter-mediated chemoresistance in small cell lung cancer. Mol Cancer Ther. 2012;11(4):830-841.

40. Nagatomo I, et al. The gefitinib-sensitizing mutant epidermal growth factor receptor enables transformation of a mouse fibroblast cell line. DNA Cell Biol. 2006;25(4):246-251.

41. Cong L, et al. Multiplex genome engineering using CRISPR/Cas systems. Science. 2013;339(6121):819-823. 